\title{
EXTRAÇÃO DE ISÓTOPOS ESTÁVEIS DE CO2 POR SUBLIMAÇÃO PARA DETERMINAÇÃO DO BALANÇO DE CARBONO DA BACIA AMAZÔNICA
}

\author{
Stéphane Palma Crispim ${ }^{1}$ \\ Lucas Gatti Domingues ${ }^{2}$ \\ Caio Silvestre de Carvalho Correia ${ }^{3}$ \\ Ricardo Santana dos Santos ${ }^{4}$ \\ Luciano Marani ${ }^{5}$ \\ Wellison Rodrigues da Costa ${ }^{6}$ \\ Luciana Vanni Gatti ${ }^{7}$
}

Resumo: Apresentamos neste trabalho a implementação de um sistema de extração de isótopos estáveis de CO2 por sublimação, que esta sendo aplicado em amostras coletadas em dois diferentes pontos Rio Branco e Alta Floresta, localidades escolhidas através de estudos para a realização do balanço de carbono na Amazônia. As coletas são feitas por meio de aviões de pequeno porte, utilizando um sistema semiautomático desenvolvido pelo GMD/ESRL/NOAA que posteriormente é analisado no laboratório localizado no Instituto Nacional de Pesquisas Espaciais em São José dos Campos e encaminhado para a realização das extrações. As amostras contendo apenas $\mathrm{CO} 2$ puro, são seladas em chama e encaminhadas para a Holanda onde serão analisados $\delta^{13} \mathrm{C}$ e $\Delta 170$.

Palavras-chave: Isótopos; Carbono; Amazônia.

\footnotetext{
${ }^{1}$ Gestão Ambiental/Instituto Nacional de Pesquisas Espaciais, Brasil. E-mail: stephane.crispim@gmail.com. 2 Mestre em Ciências Atmosféricas/Instituto Nacional de Pesquisas Espaciais, Brasil. E-mail: Igtdomingues@gmail.com.

3 Mestre em Tecnologia nuclear - Materiais/Instituto Nacional de Pesquisas Espaciais, Brasil. E-mail: cacorreia@gmail.com.

${ }^{4}$ Engenharia de Produção/Instituto Nacional de Pesquisas Espaciais, Brasil. E-mail: ricardo.inpe@gmail.com.

5 Doutor em Geofísica Espacial/Instituto Nacional de Pesquisas Espaciais, Brasil. E-mail: Imarani@gmail.com.

${ }^{6}$ Sistemas Eletrônicos/Instituto Nacional de Pesquisas Espaciais, Brasil. E-mail: wellison.ifsp@gmail.com.

${ }^{7}$ Pesquisadora Titular/Instituto Nacional de Pesquisas Espaciais, Brasil. E-mail: Ivgatti@gmail.com.
} 